\title{
The Effect of Platelet Rich Plasma (PRP) in Anterior Cruciate Ligament (ACL) Reconstruction Surgery
}

\author{
Dwikora Novembri Utomo ${ }^{1}$, Purwati ${ }^{2}$, Damayanti Tinduh ${ }^{3}$, \\ Nanang Hari Wibowo ${ }^{1}$
} ${ }^{1}$ Department of Orthopaedics and Traumatology, Faculty of Medicine, Airlangga University,
Surabaya, Indonesia

${ }^{2}$ Stem Cell Research and Development Centre, Airlangga University, Surabaya, Indonesia

${ }^{3}$ Medical Rehabilitation Department, Dr. Soetomo General Hospital, Surabaya, Indonesia

Corresponding author: dwikora_utomo@yahoo.com

Keywords: ACL reconstruction, knee joint defect, platelet rich plasma, TTDPM, JPS

\begin{abstract}
A torn in anterior cruciate ligament (ACL) caused by interference with the proprioceptive function of the knee joint can result in decreased function and other joint structure degradation that ultimately affect to daily activities. Platelet rich plasma (PRP) is widely used in recent studies to improve the healing of soft tissue injuries because it contains a lot of growth factor. This study was to determine the effect of platelet rich plasma in ACL reconstruction surgery. ACL reconstruction patients either with or without PRP were evaluated with Joint Position Sense (JPS) and Threshold To Detection of Passive Motion (TTDPM) measurements and the results were compared. From this study found 20 patients who had ACL reconstruction performed either with or without PRP. The test results showed statistically significant differences JPS in patients with ACL reconstruction who given PRP and not, at angle of $30^{\circ}(p=0,037)$ and $45^{\circ}(p=0,034)$. It also obtained a non-significant difference TTDPM in both groups $(\mathrm{p}=0,172)$. The conclusion obtained in this study is the addition of platelet rich plasma in patients with ACL reconstruction of knee joint can improve the function of JPS at an angle of $30^{\circ}$ and $45^{\circ}$, while the function of TTDPM is not increased significantly.
\end{abstract}

\section{Introduction}

Knee injury is one of the biggest problems in musculoskeletal system which is widely reported in United States with prevalence up to 48 per 1000 patients per year and from all cases of knee injury, 9\% is in the ligament where the anterior cruciate ligament (ACL) most often found [1]. Almost half of knee ligament injuries is a torn in ACL and that is the biggest causes of knee instability, which could lead to changes in function, the other joint structural damage and subsequent affects to daily activities and walking functions [2,3]. Several studies conducted in patients with torn in ACL indicated a disturbance in function of knee joint proprioception [4].

A torn in ACL can affect to the proprioception function and stability of knee joint. Most studies on knee with torn in ACL indicated decreased function of knee joint proprioception [4]. In this case, the treatment that widely used is anterior curiate ligament reconstruction, a replacement method in damaged ACL with graft. This treatment expected to restore function and improve knee joint proprioception afferent inputs which is needed for stability and joint proprioception. A histological study showed regeneration of mechano-receptors in ACL graft tissue, beginning at fourth week after reconstruction [5,6].

In another study showed motoric and sensoric behavior changes after ACL reconstruction that allegedly caused by a lack of proprioceptive information as result of ACL lesions and or ACL substitution by graft [4]. Several ACL reconstruction techniques which have been developed are expected to restore the function of knee joint. However, impaired proprioceptive function was found in mid-range motion of knee joint [7]. There is still controversy on the effects of ACL reconstruction on proprioceptive function of knee joint.

In recent years, tissue engineering has been developed and many technologies introduced in orthopedics. Gradually, the result of ACL graft showed a good increase so that shorten the healing 
time. The use of hamstring tendon autograft is preferred for several reasons such as: no risk interference in mechanism of knee extension, less anterior knee pain and less incidence of fixed flexion deformity [8]. In orthopedics and trauma surgery, using a growth factor combined with tissue engineering technique showed a good result for defect treatment $[8,9]$.

Platelet Rich Plasma (PRP) is a fraction of plasma where the platelets in plasma is concentrated [9]. Platelet rich plasma can be used in a wide variety of surgical procedures and offers many advantages. This is because its composition contains a growth factor that is unique so it can stimulate the process of proliferation, migration, and cell differentiation. PRP can be formed as a gel so it is easy to fill a particular defect. In addition, PRP-Gel can be directly injected into the bone graft or formed as a membrane to improve hemostasis and wound healing. Thus, PRP is expected to accelerate tissue regeneration $[9,10]$.

Application consensus of platelet rich plasma is not clear yet on ACL reconstruction surgery. This study aims to determine the effect of platelet rich plasma in the function of knee joint proprioception patients after ACL reconstruction.

\section{Materials and Methods}

This research was conducted using observational study design which is performed on patients. The research design is using a retrospective cohort. The evaluation performed to all samples, both treatment samples and non-treatment samples then examined the outcomes. The design as the following diagram (Figure 1) :

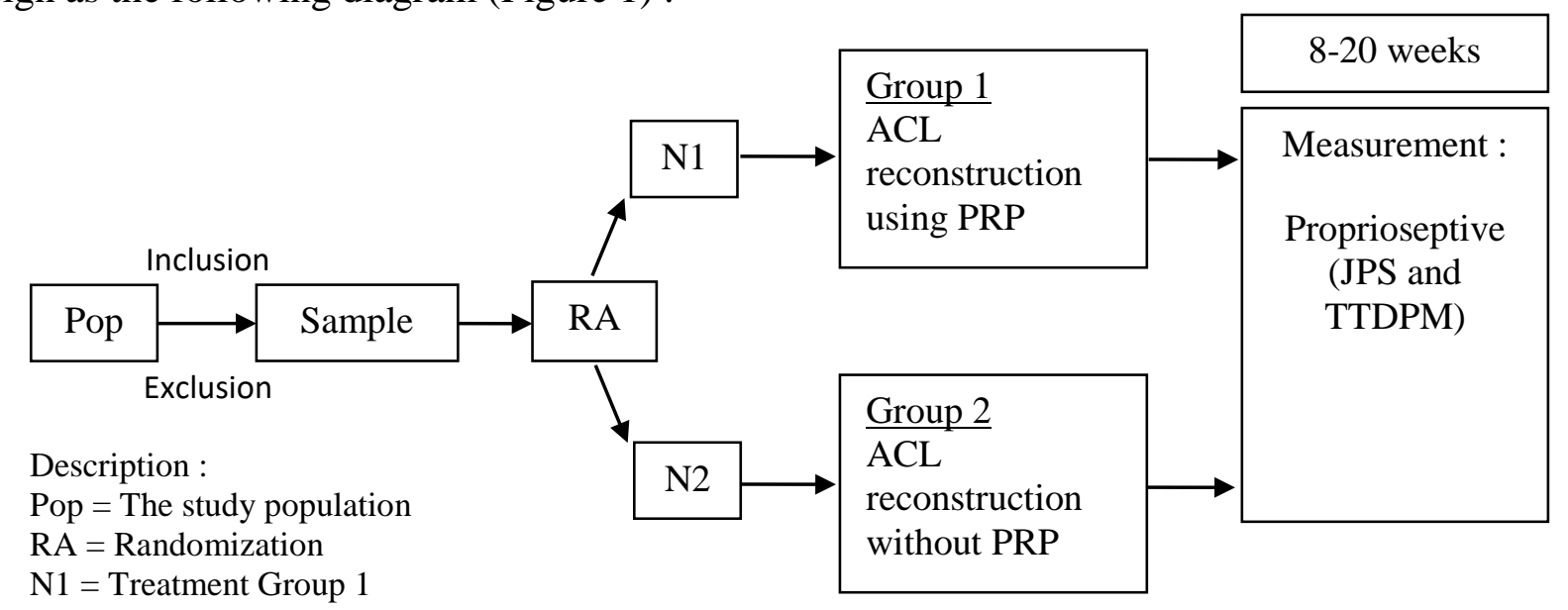

N2 $=$ Treatment Group 2

Figure 1. Observational study design diagram performed on ACL knee joint defect patients

Samples were taken with consecutive sampling from ACL reconstruction patients. Selection of the samples based on inclusion and exclusion criteria, and divided into two groups. Group 1 is given treatment with PRP and group 2 without PRP, then we measured the results using proprioceptive test. The results of two groups were then compared.

\section{Fabrication of Platelet Rich Plasma (PRP)}

Platelet rich plasma were taken from blood veins in the ear amount $10 \mathrm{cc}$ then placed into tubes containing $0,5 \mathrm{ml}$ acid citrate dextrose- $\mathrm{A}$ as an anti-coagulant and centrifuged using Kubota 6800, Tokyo, Japan machine in $1500 \mathrm{rpm}$ for 15 minutes at $40^{\circ} \mathrm{C}$. Plasma in second tube centrifuged again in $2000 \mathrm{rpm}$ for 15 minutes. Supernatant from second centrifuge discarded and produce approximately $0,8 \mathrm{ml}$ of PRP (about $10 \%$ ).

\section{Platelet Rich Plasma Administration Protocol}

After blood's patients were taken from peripheral to produce PRP, patients were then carried out reconstruction by injecting PRP into knee joint, especially focusing on entrance and exit of ACL as shown in Figure 2. 


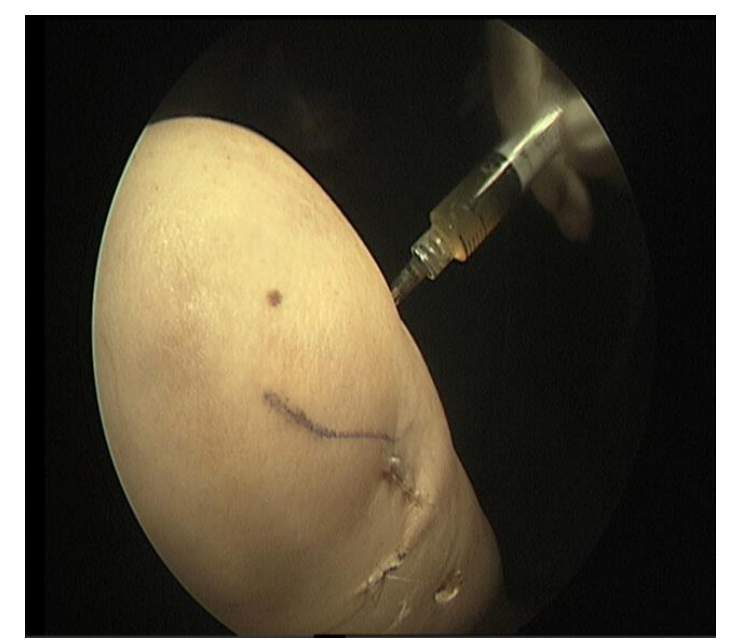

Figure 2. PRP administration into the knee joint

\section{Proprioceptive Test}

Proprioceptive test is used to determine the knee function by measuring Joint Position Sense (JPS) and Threshold To Detection of Passive Motion (TTDPM) using isokinetic dynamometer with eyes and ears closed to reduce the movement of knee joint from vision and hearing (the sound of engine dynamometer). JPS measurement performed at an angle of $30^{\circ}, 45^{\circ}$ and $60^{\circ}$, while TTDPM at a speed of $1 \% \mathrm{sec}$. The average taken from each subject in three times measurements. JPS average calculated from the difference reproduction error predetermined angle.

\section{Results}

The purpose of statistical analysis in this study is to compare the test results between JPS and TTDPM on healthy knee and ACL knee reconstruction. Data should be normally distributed to compare the results between JPS and TTDPM from ACL knee reconstruction with and without PRP. We used Parameterik test which is Independent T test. The terms of using this test is all the data group should be normally distributed. Homogeneity test of data is using Shapiro-Wilk test.

The results of statistical test analysis using Kolmogorov-Smirnov and Shapiro-Wilk in patients with a healthy knee and ACL knee reconstruction at JPS angle of $30^{\circ}, 45^{\circ}$ and $60^{\circ}$, TTDPM at speed of $1^{\circ} / \mathrm{sec}$, also treatment with and without PRP were shown in Table 1 . These results indicated that there is a significant difference statistically in JPS angle of $30^{\circ}$ and $45^{\circ}$ between treatment and control group (healthy knees), while not found significant differences in JPS angle of $60^{\circ}$ and TTDPM when compared to control group.

Table 1 . Test of normality

\begin{tabular}{|l|l|r|r|r|r|r|r|}
\hline \multirow{2}{*}{ Group } & \multicolumn{2}{|c|}{ Kolmogorov-Smirnov $^{\mathrm{a}}$} & \multicolumn{3}{|c|}{ Shapiro-Wilk } \\
\cline { 3 - 8 } & Statistic & df & Sig. & Statistic & df & Sig. \\
\hline \multirow{2}{*}{ JPS 30 T } & PRP - &, 206 & 8 &, $200^{*}$ &, 890 & 8 &, 232 \\
\cline { 2 - 8 } & PRP + &, 160 & 12 &, $200^{*}$ &, 956 & 12 &, 729 \\
\hline \multirow{2}{*}{ JPS 30 C } & PRP - &, 157 & 8 &, $200^{*}$ &, 928 & 8 &, 496 \\
\cline { 2 - 9 } & PRP + &, 151 & 12 &, $200^{*}$ &, 939 & 12 &, 481 \\
\hline \multirow{2}{*}{ JPS 45 T } & PRP - &, 154 & 8 &, $200^{*}$ &, 936 & 8 &, 577 \\
\cline { 2 - 8 } & PRP + &, 236 & 12 &, 064 &, 904 & 12 &, 178 \\
\hline JPS 45 C & PRP - &, 210 & 8 &, $200^{*}$ &, 943 & 8 &, 642 \\
\hline
\end{tabular}




\begin{tabular}{|l|l|r|r|r|r|r|r|}
\hline & PRP + &, 174 & 12 &, $200^{*}$ &, 937 & 12 &, 458 \\
\hline \multirow{2}{*}{ JPS 60 T } & PRP - &, 219 & 8 &, $200^{*}$ &, 921 & 8 &, 434 \\
\cline { 2 - 8 } & PRP + &, 225 & 12 &, 095 &, 860 & 12 &, 049 \\
\hline \multirow{2}{*}{ JPS 60 C } & PRP - &, 149 & 8 &, $200^{*}$ &, 945 & 8 &, 657 \\
\cline { 2 - 8 } & PRP + &, 228 & 12 &, 086 &, 899 & 12 &, 155 \\
\hline \multirow{2}{*}{ TTDPM T } & PRP - &, 220 & 8 &, $200^{*}$ &, 930 & 8 &, 520 \\
\cline { 2 - 8 } & PRP + &, 153 & 12 &, $200^{*}$ &, 905 & 12 &, 182 \\
\hline \multirow{2}{*}{ TTDPM C } & PRP - &, 188 & 8 &, $200^{*}$ &, 944 & 8 &, 651 \\
\cline { 2 - 8 } & PRP + &, 165 & 12 &, $200^{*}$ &, 858 & 12 &, 047 \\
\hline
\end{tabular}

*. This is a lower bound of the true significance.

a. Lilliefors Significance Correction

T. Treatment group, C. Control group

\section{Discussion}

\section{The Study Characteristics}

This study was conducted on 20 patients with torn in ACL reconstruction surgery between 8 to 20 months. Patients were divided into two groups with 12 patients given PRP and 8 patients without PRP. Most patients were in 20-30 years old, while the rest in 31-35 years old.

The examination of proprioceptive function of knee joint in ACL patients is mostly performed using JPS and or TTDPM methods, although there is still no standard procedure approved such as angles, angular velocity, position checking, tools used and time of examination. In this study, the angle used for JPS examinations are at $30^{\circ}, 45^{\circ}$ and $60^{\circ}$ based on the anatomy and biomechanics of ACL which is stabilizing the movement of knee joint during flexion (bundle anteromedial) and extension (bundle posterolateral) and the angle of knee when daily activities. TTDPM examination conducted by the angular velocity of $1 \% \mathrm{sec}$ to stimulate joint receptors without stimulating muscle receptors.

Study by Shimizu reported that regeneration of mechano-receptors on ACL graft arise during the period of at least two to four weeks post-surgery up to maximum of eight weeks post surgery [6]. In the postoperative period of six weeks, there has been a formation of fibroblasts and collagen in ACL. Type of reconstruction performed may affect the improvement of knee joint proprioception. In this study, the type of reconstruction has been controlled by the same type of ACL reconstruction on all subjects.

\section{JPS and TTDPM Measurement Results}

These measurements results indicated that there is a significant difference statistically in JPS angle of $30^{\circ}$ and $45^{\circ}$ between treatment group and control group (healthy knees), while not found significant differences in JPS angle of $60^{\circ}$ and TTDPM when compared to control group.

This is consistent with several studies conducted in subjects with ACL reconstruction. Bonfim concluded that there are still proprioceptive deficits in subjects after ACL reconstruction in period between 12 to 30 months postoperative [3]. Mou-wang reported JPS deficits in subjects after ACL reconstruction in period of 6 months postoperative [11]. Fremerey reported that there are still deficits of JPS in 3 months postoperative in knee extension position $\left(0^{\circ}-20^{\circ}\right)$, mid-range $\left(40^{\circ}-60^{\circ}\right)$ and flexion $\left(80^{\circ}-100^{\circ}\right)$, and there are still deficits of JPS mid-range position until three years after reconstruction [7].

Anterior cruciate ligament damage lead to functional disorders that contribute to control knee movement and impaired proprioceptive function of knee joint caused by reduced 
mechanoreceptors that play a role in sensory input regarding the position sense (especially on the position of extension) and a sense of joint movement [12]. Friden examined the knee joint proprioception in 19 healthy subjects and 20 subjects with impaired ACL one side and concluded that reproduction actively positioning the knee joint flexion at $30^{\circ}-60^{\circ}$ is more accurate when compared to the position angle of extension at $60^{\circ}-30^{\circ}$ [13]. This is related to the ACL protective function of knee against excessive movement, especially on the position of knee joint extension. Another study concluded that ACL strain will appear at the quadriceps muscle to moves tibia towards full extension. The ability of quadriceps muscle to stretch ACL in full extension position because this position maximizes the patellar tendon insertion angle relative to tibia thus causing the translation of tibia anteriorly.

This study does not correspond to study by Hopper, who reported that there is no interference proprioceptive function of knee joint in patients after ACL reconstruction in period of 12 to 16 months postoperative measured by JPS in standing position (weight bearing) [14]. This can be caused by differences in measurement time period in which at month 12 to 16 postoperative regeneration has occurred perfect mechanoreceptors. In addition, the method used for measurement in JPS standing position will provide sensory feedback of extra-articular structures that help determine joint position more accurate [11].

Statistically significant differences were found in JPS and TTDPM knee in group that received PRP when compared with JPS and TTDPM in group that did not receive PRP at an angle of $30^{\circ}$ and $45^{\circ}$. There is no significant difference was found between JPS at an angle of $60^{\circ}$ with TTDPM. These results are expected because PRP contains many unique growth factor that stimulates proliferation, migration and differentiation of cells physically.

According to the study of Nather, PRP can be used in a wide variety of surgical procedures and offers many advantages. PRP gel can be directly injected into the bone graft or can formed as membrane structure so that it can be put on field operations to improve hemostasis and healing. In ultrastructure, fibrin holes serve as scaffold for migration and stimulate hemostasis and tissue adhesion, but at the same time, the holes provide new vascular growth opportunities and ensure oxygenation [15].

TTDPM average in both groups showed no significant difference. There are two things that can explain this. First, the most population of mechanoreceptors in ACL is Ruffini endings which play an important role in stimulation of joint positions both static and dynamic. Population Pacini as mechanoreceptors which lead to dynamic movement of joint less than Ruffini endings [16]. Pacini regeneration post-reconstruction is faster than Ruffini endings [17]. This causes post-reconstruction TTDPM or kinesthesia not disrupted, although the population before injury less than Ruffini. Second, TTDPM examination in this study using Cybex machine Isokinetic dynamometer which in its regulation must be moved first in flexion direction of knee before moving the machine itself, so that the subjects know when the machine begins to move, and this led to biases examination.

The results of this study provide information about PRP advantages to proprioception function in patient's knee after ACL reconstruction. PRP provides improvements to JPS function, although not to the TTDPM.

\section{Conclusion}

The conclusion obtained in this study is provision of platelet rich plasma (PRP) in patients with reconstruction of anterior cruciate ligament (ACL) of knee joint can improve function joint position sense (JPS) at an angle of $30^{\circ}$ and $45^{\circ}$, while the function of the threshold to detection of passive motion (TTDPM) did not increase significantly. 


\section{References}

[1] U.G. Longo, J.B. King, V. Denaro, N. Maffulli. 2008. Double Bundle Arthroscopic Reconstruction of The Anterior Cruciate Ligament. J Bone Joint Surg. 90-B: 995-9.

[2] DeFranco M.J, Bernard R. Bach J. 2009. A Comprehensive Review of Partial Anterior Cruciate Ligament Tears. J Bone Joint Surg Am. 91:198-208.

[3] Bonfim T.R, Paccola C.A.J, Barela J.A. 2003. Proprioceptive and Behavior Impairments in Individuals With Anterior Cruciate Ligament Reconstructed Knees. Arch Phys Med Rehabil. 84:1217-23.

[4] Pap G, Machner A, Nebelung W, Awiszus F. 1999. Detailed analysis of proprioception in normal and ACL-deficient knees. J Bone Joint Surg [Br];81.

[5] Schultz R.A, Miller D.C, Kerri C.S, Micheli L. 1984. Mechanoreceptors in Human Cruciate Ligaments. A Histological Study. J Bone Joint Surg Am. 66(7):1072-6.

[6] Shimizu T, Takahashi T, Wada Y, Tanaka M, Morisawa Y, Yamamoto H. 1999. Regeneration Process of Mechanoreceptors in The Reconstructed Anterior Cruciate Ligament. Arch Orthop Trauma Surg. [Original Article]. 119:405-9.

[7] Fremerey R.W, Lobenhoffer P, Zeichen J, Skutek M, Bosch U, Tscherne H. 2000. Proprioception After Rehabilitation and Reconstruction in Knees with Deficiency of The Anterior Cruciate Ligament. British Editorial Society of Bone and Joint Surgery. [A Prospective, Longitudinal Study]. 82-B (6):801-6.

[8] Ferretti M, Zelle B.A, Chhabra A. 2005. Anatomy Anterior Cruciate Ligament DoubleBundle Reconstruction Using 2 Tibial and 2 Femoral Tunnel. Techniques in Orthopaedics. 20(3):218-223.

[9] Louay Fallouh, Koichi Nakagawa, Takahisa Sasho. 2010. Effects of Autologous PlateletRich Plasma on Cell Viability and Collagen Synthesis in Injured Human Anterior Cruciate Ligament. J Bone Joint Surg Am. 92:2909-16.

[10] Taylor D.W, Petrera M, Hendry M, Theodoropoulos J.S. 2011. A Systematic Review of The Use of Platelet-Rich Plasma in Sports Medicine as A New Treatment for Tendon. Clin J Sport Med. 21:344-352.

[11] Mou-wang Z, Li G, Ya-ping C, Chang-long Y, Ying-fang A, Hong-shi H. 2008. Factors Affecting Proprioceptive Recovery After Anterior Cruciate Ligament Reconstruction. Chin Med;121(22):2224-8.

[12] Fu Freddie H, Harner C.D. 1993. Biomechanics of Knee Ligament : Basic Concept and Clinical Application. J Bone Joint Surg (Am). 75:1716-1727.

[13] Friden T, Roberts D, Zaitterstrom R, Lindstrand A, Moritz U. 1996. Proprioception in The Nearly Extended Knee: Measurements of Position and Movement in Healthy Individuals and in Symptomatic Anterior Cruciate Ligament Injured Patients. Knee Surg, Sports Traumatol, Arthroscopy. 4:217-24.

[14] Frank C.B, Jackson D.W. 1997. Current Concept Review-The Science of Reconstructon of The Anterior Cruciate Ligament. J Bone Joint Surg (Am). p. 79:1556-76.

[15] Nather A, Manohara R, De S.D. 2005. Platelet Rich Plasma in Orthopedics Surgery: Basic Science and Clinical Application. Bone Graft and Bone Substitute Basic Science and Clinical Application. Singapore.

[16] Beard D.J, Kyberd P.J, Fergusson C.M, Dodd C.A.F. 1993. Proprioception After Rupture of The Anterior Cruciate Ligament: An Objective Indication of The Need for Surgery. J Bone Joint Surg [Br].75-B:311-5.

[17] Neumann D. Knee. In: Neumann D, Editor. 2002. Kinesiology of The Musculoskeletal System. St. Louis Missouri: Mosby. p. 434-76.

[18] Nagai T, Sell T.C, Lephart S.M. 2007. Effect of Age and Osteoarthritis on Knee Proprioception. US Musculoskeletal Review. p. 69-70.

[19] Anders J.O, Venbrocks R.A, Weinberg M. 2008. Proprioceptive Skills and Functional Outcome After Anterior Cruciate Ligament Reconstruction with Bone-Tendon-Bone Graft. International Orthopaedics. [Original paper]. 32:627-33. 